\title{
Perfil de los usuarios de una unidad móvil de drogodependencias en el sureste de España
}

\author{
Consuelo Egea Zapata*; M ${ }^{a}$ Dolores Pérez Cárceles**; Eduardo Osuna Carrillo de Albornoz**; María Falcón \\ Romero***; Aurelio Luna Maldonado** \\ * Médico. Unidad Móvil de Drogodependencias. Murcia. \\ * Médicos. Área de Medicina Legal y Forense. Universidad de Murcia. \\ *** Farmacéutica. Área de Medicina Legal y Forense. Universidad de Murcia \\ Enviar correspondencia a: \\ Ma Dolores Pérez Cárceles. Facultad de Medicina. Area de Medicina Legal y Forense. Universidad de Murcia. Campus de Espinardo. 30100 Murcia.
} Telf: 968/36 7018. Fax: 968/36 4150. E-Mail: mdperez@um.es

Recibido: 7 de octubre de 2004. Aceptado: 26 de septiembre de 2005.

\section{RESUMEN}

Introducción. Los programas de mantenimiento con metadona se enmarcan dentro de la filosofía de reducción del daño, destinados a mejorar la salud y la calidad de vida de los consumidores de drogas que no quieren o no pueden abandonar su adicción. Los objetivos de este trabajo son conocer el perfil de los sujetos adictos a la heroína cuando inician la demanda de tratamiento en un programa de mantenimiento con metadona, conocer si existen diferencias con respecto a los usuarios de otros recursos y la aceptación de la Unidad Móvil. Material y métodos. Estudio descriptivo, transversal, basado en un cuestionario a 135 sujetos, seleccionados mediante muestreo aleatorio estratificado, de entre todos los sujetos que demandan tratamiento por primera vez en una Unidad Móvil de Drogodependencias. Las variables analizadas han sido: características sociodemográficas, familiares, personales, de patrones de consumo, clínicas y relacionadas con la utilización de la Unidad Móvil. Resultados. Los sujetos son mayoritariamente hombres jóvenes (edad media= 26,2 \pm 4,6 años), la edad media de abandono de los estudios es de 14,4 $\pm 2,3$ años, habiendo sido detenidos en alguna ocasión el 74,1\%. Consumen diariamente heroína, cocaína, benzodiacepinas, cannabis, alcohol y tabaco el 100\%,64\%, 18\%, 19\%, $58 \%$, y $98 \%$ de los sujetos, respectivamente. Más de un tercio de estos sujetos desconocían su estado serológico. El 37,8\% de los sujetos no habían realizado tratamientos previos. En el $90 \%$ de los casos los sujetos refieren que la persona que les informó de las características y uso de la unidad móvil son sujetos que han utilizado también este recurso o lo siguen haciendo. Conclusión. El perfil de esta población viene definido por un bajo nivel socio-cultural y alto nivel de marginación, así como por un consumo precoz del alcohol, tabaco y cannabis. La unidad móvil de drogodependencias permite contactar con un grupo de población drogodependiente que no se había visto motivada para el tratamiento y que habitualmente se mantiene al margen de otros servicios asistenciales. La influencia de los pares para iniciar el tratamiento es elevada.

Palabras-clave: metadona, consumo de drogas, unidad móvil.

\section{ABSTRACT}

Introduction. Metadone maintenance programmes are about harm reduction, as understood by a series of measures destined to improve the health and quality of life of those who do not want or who cannot give up their addiction. The aim of this paper is to describe the profile of heroin addict when they first sought treatment in a Mobile Drug dependency Unit (MDU), and their acceptance of the unit. Material and methods. Questionnaire-based, descriptive, transversal study of 135 subjects selected in a stratified random way from subjects seeking treatment for the first time in an MDU in Murcia (SE Spain). Details of socio-demographic characteristics, family, personal, consumption, and attendance at therapeutic programmes, clinics and the mobile unit itself were included. Results. The users were mostly young men with a mean age of $26.2 \pm 4.6$ years. The average school leaving age was $14.4 \pm 2.3$ years, and $74.1 \%$ had been detained previously by the police. The proportion of daily consumption among users for heroin, cocaine, benzodiacepines, cannabis, alcohol and tobacco was $100 \%, 64 \%, 18 \%, 19 \%, 58 \%$, and $98 \%$, respectively. $37.8 \%$ subjects had not received treatment previously. More than a third of users had no knowledge of their serological state. In $90 \%$ of cases, the person suggesting that the interviewed subject attended the MDU had himself used the service and continued doing so. Conclusion. The profile of the population seeking advice is defined by their low socio-cultural status, social alienation and early use of alcohol, tobacco and cannabis. The MDU was able to establish contact with a group from the drug-dependent population which had not been motivated to seek treatment before and which would normally shy away from other social services. The influence of partners on beginning treatment was high.

Keywords: methadone, substance abuse, mobile unit. 


\section{INTRODUCCIÓN}

L a adicción a sustancias constituye uno de los principales problemas de salud en el momento actual. En España el uso de drogas ilegales continúa siendo una causa importante de mortalidad juvenil'. El consumo de drogas, especialmente por vía parenteral, implica numerosos riesgos para la salud individual y colectiva, principalmente la transmisión de enfermedades como el $\mathrm{VIH}$, hepatitis B y hepatitis $\mathrm{C}$, por lo que su prevención y corrección se convierten en objetivo sanitario de primer orden. Mejorar los niveles de salud entre los consumidores y controlar la propagación que a partir de ellos pueda efectuarse es la exigencia más urgente en el tratamiento de los programas asociados al uso de drogas. Aceijas et al.. ${ }^{2}$ refieren prevalencias por encima del $20 \%$ del VIH entre los usuarios de drogas por vía parenteral en zonas de 25 países, entre los que se encuentra España.

La abstinencia ha dejado de ser el único objetivo a conseguir y los programas de tratamiento no se centran únicamente en el tratamiento de la drogodependencia en sí misma, sino que se van a ir configurando programas enmarcados dentro de la denominada filosofía de reducción del daño, entendida como el conjunto de acciones destinadas a mejorar la salud y la calidad de vida de los consumidores de drogas que no quieren o no pueden abandonar su adicción. Recientemente Hope et al.. ${ }^{3}$ señalan la necesidad de reimpulsar las medidas de reducción del daño y adaptarlas a los comportamientos de riesgo en el consumo de drogas.

Las intervenciones para afrontar dichos daños incluyen: el cuidado de la salud, que incluye el tratamiento de los trastornos por uso de sustancias de los actuales usuarios, la reducción del número de personas que están empezando a utilizar drogas y facilitar a los usuarios de drogas formas más seguras de uso. Dichas intervenciones se pueden llevar a cabo desde centros fijos o bien desde Unidades Móviles.

La prescripción de metadona es sólo uno de los componentes de los programas de mantenimiento con metadona, y ésta ha de ir acompañada de una intervención sanitaria, psicológica y social ${ }^{4,5}$. La efectividad de dichos programas en términos de reducción del daño, reducción de la actividad delictiva e incremento de la integración socio-laboral está ampliamente documentada ${ }^{6}$.

Las Unidades Móviles de prescripción y dispensación de metadona acercan los recursos sanitarios a colectivos sociales que se caracterizan, habitualmente, por pertenecer a zonas especialmente deprimidas y marginadas de las ciudades y que presentan una motivación más que limitada para acudir a los Centros de Atención a Drogodependientes. En la ciudad de
Murcia surge en 1993 una de estas Unidades Móviles con el objetivo principal de acercarse a los usuarios de drogas que no contactaban con los centros ambulatorios. En esta unidad se lleva a cabo un programa de tratamiento de bajo umbral o exigencia, siendo los objetivos del tratamiento admitir y mantener en tratamiento sustitutivo con metadona el máximo número de pacientes dependientes de opiáceos. Además de complementarlo un programa de intercambio de jeringuillas, accesibilidad a preservativos y realización de analíticas para la detección de VIH, hepatitis B y C, sífilis y tuberculosis.

Debido a la generalización en España durante los últimos años de este tipo de recursos es necesario conocer el perfil de la población que los utiliza. Los objetivos de este trabajo son analizar las características de los usuarios de una Unidad Móvil de Drogodependencias, conocer si existen diferencias con respecto a los usuarios de otros recursos y la aceptación de este recurso por parte de los usuarios.

\section{MATERIAL Y MÉTODOS}

Estudio descriptivo transversal. Se realizó un muestreo aleatorio tomando como universo muestral el total de los pacientes $(N=549)$ que acudieron demandando tratamiento directamente a la UMD durante 2 años (Agosto 1998-2000). La muestra estudiada fueron 135 usuarios que acudieron demandando tratamiento por primera vez a una Unidad Móvil de Drogodependencias (UMD), seleccionados aleatoriamente por el médico de la unidad móvil que realizó las encuestas. La muestra está formada por 119 hombres $(88,1 \%)$ y 16 mujeres $(11,9 \%)$, con una edad media de 26,2 (ds: 4,6 años; rango 17-38 años).

La Unidad Móvil está dotada de dos médicos y dos guardias de seguridad que realiza de lunes a viernes, en turno de mañana y tarde, un mismo recorrido por 25 puntos del Área de Salud I de la Región de Murcia. La petición para ser incluido en el Programa de mantenimiento con Metadona de la UMD la solicita el usuario directamente en la unidad móvil.

La recogida de datos se realizó mediante una entrevista personal, voluntariamente aceptada por el paciente, en el momento de realizar éste por primera vez la demanda de tratamiento en la propia Unidad Móvil. Acudir a la UMD era la primera demanda de tratamiento para 51 usuarios (37,8\%). Se les garantizó la confidencialidad de la información no recogiendo datos de identificación del paciente en los cuestionarios. A cada paciente se le informó de los objetivos del estudio y se solicitó su consentimiento para formar parte de él. La entrevista fue realizada en todos 
los casos por el médico de la Unidad Móvil, teniendo una duración aproximada de 15 minutos.

El cuestionario recoge diferentes tipos de variables: Sociodemográficas, familiares, personales, relacionadas con los patrones de consumo, asistenciales y terapéuticas de la dependencia a tóxicos, clínicas, y relacionadas con la utilización de la UMD.

Para el análisis estadístico se utilizó el programa SPSS 11.0. Se realizó un análisis descriptivo de la muestra. Las variables cuantitativas distribuidas simétricamente fueron descritas utilizando la media y la desviación estándar. La comparación de variables cualitativas se realizó mediante la prueba de la $\chi^{2}$ de Pearson. Utilizamos este test para conocer si existe una asociación estadísticamente significativa entre la edad (distribuida en grupos) de inicio en el consumo de opiáceos y la vía de consumo, entre la etnia y las características de los usuarios, en los cambios higiénicos en el uso de la jeringuilla desde el comienzo del consumo hasta que demandan tratamiento en la UMD y si existían diferencias significativas entre los usuarios del Centro de Atención al Drogodependiente (CAD) y los de la UMD. Se rechazó la hipótesis nula para valores de $p$ inferiores a 0,05.

\section{RESULTADOS}

La tabla 1 recoge el perfil del usuario al iniciar tratamiento en la Unidad Móvil de Drogodependencias.

\section{Características sociodemográficas}

En nuestra muestra predominan los sujetos solte$\operatorname{ros}(61,6 \%)$ que viven en el domicilio familiar $(79,2 \%)$, siendo mayoritarias las familias numerosas, con un número medio de hermanos de 5,3 $\pm 2,6$. El $20 \%$ de los sujetos son de etnia gitana y el perfil de estos sujetos aparece en la tabla 2.

Las relaciones familiares eran consideradas como buenas en el $47,4 \%$ de los usuarios, estando deterioradas en el resto de los casos y siendo el motivo principal de este deterioro el consumo de drogas (80,9\%).

En cuanto al nivel educativo de los usuarios tan sólo el 27,4\% de ellos finalizó los estudios primarios, siendo la edad media de abandono de los estudios de 14,4 \pm 2,3 años. En el 34,1\% de los casos los sujetos refieren haber sido expulsados del colegio en alguna ocasión, siendo los motivos principales para la expulsión el mal comportamiento (36,9\%) seguido del absentismo escolar $(23,9 \%)$ y violencia en las aulas $(21,7 \%)$. La edad media de inicio laboral de estos pacientes fue de 14,6 \pm 2,2 años (rango 8-21), siendo la

\section{Tabla 1. Características sociodemográficas y de consumo de heroína}

\begin{tabular}{|c|c|}
\hline Edad, media (ds) años & $26,2(4,6)$ \\
\hline Varones (\%) & 88,1 \\
\hline \multicolumn{2}{|l|}{ Estado civil (\%) } \\
\hline Casados & 14,8 \\
\hline Solteros & 61,6 \\
\hline Separados & 8,1 \\
\hline Pareja de hecho & 15,5 \\
\hline \multicolumn{2}{|l|}{ Convivencia (\%) } \\
\hline Cónyuge/pareja & 9,6 \\
\hline Familia ascendente & 61,5 \\
\hline Familia propia+ascendente & 17,8 \\
\hline Amigos & 3,7 \\
\hline Solo & 7,4 \\
\hline \multicolumn{2}{|l|}{ Estudios (\%) } \\
\hline Analfabeto & 5,9 \\
\hline Analfabeto funcional & 6,7 \\
\hline Primarios incompletos & 57,1 \\
\hline Primarios completos & 27,4 \\
\hline Medios & 2,9 \\
\hline Superiores & 0 \\
\hline \multicolumn{2}{|l|}{ Situación laboral (\%) } \\
\hline Contratado fijo & 3,7 \\
\hline Contratado eventual & 3,7 \\
\hline Parado & 91,1 \\
\hline Pensionista & 1,5 \\
\hline Antecedentes delictivos (\%) & 74,1 \\
\hline Antecedentes encarcelamiento (\%) & 56,3 \\
\hline
\end{tabular}

Edad inicio consumo heroína,

media (ds) años

$18,1(3,8)$

Vía de administración heroína (\%) 65,6 intravenosa

Droga secundaria

Cannabis (\%) 75,6

Cocaína (\%) 74

$\mathrm{VHB}+(\%) \quad 18,5$

$\mathrm{VHC}+(\%) \quad 37,8$

$\mathrm{VIH}+(\%) \quad 15,6$

Tratamientos previos por dependencia

a heroína (\%)

62,2

actividad laboral más frecuentemente realizada actividades en la rama de la construcción (19,3\%).

\section{Conductas antisociales}

En lo que respecta a antecedentes delictivos habían tenido contacto con el Juzgado de Menores 40 de los sujetos $(29,6 \%)$, siendo el motivo principal por el que habían sido detenidos siendo menores de 18 años, el robo (45\%). La edad media de la primera detención es de $17 \pm 4,1$ años, presentando antecedentes delictivos el $74,1 \%$ de los usuarios. En los doce 
Tabla 2. Diferencias estadísticamente significativas entre el perfil del usuario de etnia gitana y el resto de usuarios de la UMD.

\begin{tabular}{lccc}
\hline & $\begin{array}{c}\text { Etnia gitana } \\
\mathbf{N = 2 7}\end{array}$ & $\begin{array}{c}\text { No gitana } \\
\mathbf{N = 1 0 8}\end{array}$ & $\mathbf{P}$ \\
\hline Analfabetos & $48,1 \%$ & $29,6 \%$ & $<0,001$ \\
Casados o conviviendo con pareja estable & $59,2 \%$ & $14,8 \%$ & 0,02 \\
Domicilio estable & $33,3 \%$ & $64,6 \%$ & 0,008 \\
Ingreso en centros de menores & $14,8 \%$ & $5,2 \%$ & 0,04 \\
Vía de administración de la heróna inhalada/fumada & $48,1 \%$ & $29,8 \%$ & 0,03 \\
Gasto en el consumo de heroína & $54,04 € /$ día & $35 € /$ día & $<0,0001$ \\
VHB + & $7,4 \%$ & $18,5 \%$ & 0,03 \\
VHC + & $11,1 \%$ & $37,8 \%$ & 0,03 \\
VIH + & $7,4 \%$ & $15,6 \%$ & 0,04 \\
Nunca se han realizado analíticas & $81,5 \%$ & $27,8 \%$ & $<0,001$ \\
No tratamientos previos por dependencia a la heroína & $81,5 \%$ & $27,8 \%$ & 0,01 \\
Información de la UMD & $40,7 \%$ & $52,6 \%$ & 0,01 \\
& informados por & informados por & amigos \\
familiares & \multicolumn{3}{c}{} \\
P= probabilidad; $\chi^{2}$ de Pearson & \multicolumn{3}{c}{} \\
\hline
\end{tabular}

meses previos a la entrevista habían sido detenidos 40 sujetos $(29,6 \%)$, siendo también el motivo principal el robo. Se encontraban pendiente de algún juicio 46 individuos $(34,1 \%)$. El $56,3 \%$ de los usuarios habían estado ingresados en algún centro penitenciario y en el año previo a la entrevista habían ingresado en prisión en alguna ocasión el 31,9\% de ellos.

\section{Perfil de consumo}

En el momento de realizarse la entrevista la vía usada con mayor frecuencia para el consumo de heroína es la intravenosa $(65,9 \%)$, gastando el paciente en el consumo de heroína una media de 34,39 $\pm 22,26$ $€ /$ día. La media del número de administraciones fue de 4,0 $\pm 2,0$ veces/día, prefiriendo a la hora de consumir estar solo en el $43,4 \%$ de los casos. En cuanto a los patrones higiénicos en el uso de jeringuillas para el consumo durante el curso de su adicción (desde el inicio hasta el momento de realizarse la entrevista) se observa un cambio en las conductas de riesgo, predominando en el inicio del consumo el hecho de compartir jeringuillas con diferentes personas $(37 \%)$ y en el momento de realizarse la entrevista el $50,4 \%$ de los usuarios utilizaba una jeringuilla para administrarse varias dosis, pero sin compartirlas con otros usuarios. Los hábitos higiénicos en relación con el uso de las jeringuillas cuando los sujetos comienzan el consumo de opiáceos y en el momento de realizar la entrevista aparece en la tabla 3.

Tabla 3. Cambios higiénicos en el uso de la jeringuilla desde el comienzo del consumo hasta que demandan tratamiento en la UMD.

\begin{tabular}{|c|c|c|c|c|c|}
\hline \multirow[t]{2}{*}{ Uso de la jeringuilla } & \multicolumn{2}{|c|}{ Inicio en el consumo } & \multicolumn{2}{|c|}{ Demanda de tratamiento en la UMD } & \multirow{2}{*}{$\mathbf{P}$} \\
\hline & $\mathbf{N}$ & $\%$ & $\mathbf{N}$ & $\%$ & \\
\hline No vía intravenosa & 26 & 19,3 & 40 & 29,6 & 0,03 \\
\hline Una jeringuilla por dosis & 14 & 10,4 & 19 & 14,1 & 0,04 \\
\hline Una jeringuilla para varias dosis & 27 & 20 & 68 & 50,4 & 0,01 \\
\hline Compartida con la misma persona & 18 & 13,3 & 3 & 2,2 & 0,04 \\
\hline Compartida con diferentes personas & 50 & 37 & 5 & 3,7 & 0,02 \\
\hline De procedencia desconocida & 0 & 0 & 0 & 0 & NS \\
\hline Total & 135 & 100 & 135 & 100 & \\
\hline
\end{tabular}


Tabla 4. Perfil de los usuarios en la primera demanda de tratamiento en el CAD y en la UMD.

\begin{tabular}{lccc}
\hline Primera demanda de tratamiento & CAD & UMD & P \\
\hline Varones (\%) & 89,7 & 88,1 & NS \\
Edad media (ds) & $26,3 \pm 3,2$ & $26,2 \pm 4,6$ & NS \\
Edad de inicio en el consumo de opiáceos (ds) & $21,2(3,6)$ & $18,3(2,5)$ & 0,038 \\
Años de consumo (media, ds) & $5,2(2,1)$ & $8(2,4)$ & 0,021 \\
Consumo de sustancias en los 30 días anteriores: & & & \\
$\quad$ Cannabis (\%) & 36,2 & 75,6 & 0,022 \\
$\quad$ Cocaína (\%) & 42,6 & 74 & 0,034 \\
$\quad$ Benzodiacepinas (\%) & 17,2 & 55,4 & 0,027 \\
$\quad$ Anfetaminas (\%) & 3,3 & 5,1 & NS \\
Vía principal consumo de heroína: & 56,5 & 28,1 & 0,026 \\
$\quad$ Fumada/Inhalada (\%) & 39 & 65,6 & 0,03 \\
$\quad$ Intravenosa (\%) & 3,6 & 1,5 & 0,04 \\
$\quad$ Esnifada (\%) & 11,8 & 15,6 & $\mathrm{NS}$ \\
VIH + (\%) & & & \\
\hline
\end{tabular}

$\mathrm{P}=$ probabilidad; $\chi^{2}$ de Pearson; $\mathrm{NS}=$ no significativo

Hemos de destacar que existe una relación significativa $(P=0,0001)$ entre la edad de los pacientes en el momento de la entrevista y la vía de inicio en el consumo de opiáceos. Así los pacientes menores de 20 años la vía de inicio en el consumo es la fumada/inhalada $(68,7 \%)$, mientras que en los mayores de 25 la vía principal al inicio es la intravenosa (73,8\%).

Habían seguido tratamientos previos a la entrevista en la Unidad Móvil, 84 usuarios (62,2\%) siendo el tratamiento realizado con más frecuencia un Programa de Mantenimiento con Metadona (28,8\%). El hecho más destacado de estos tratamientos era su abandono precoz, siendo el motivo principal para abandonar el tratamiento iniciado seguir consumiendo heroína (42,9\%).

En cuanto a la frecuencia en el consumo de otras sustancias destaca el consumo diario de cocaína $(64,6 \%)$, cannabis $(19,3 \%)$ y benzodiacepinas $(17,8 \%)$. El 98,5\% de los pacientes fuma, siendo el número

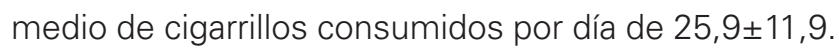
El $57,8 \%$ de los sujetos es consumidor de bebidas alcohólicas siendo el consumo medio de gramos de alcohol en día laborales $66,7 \pm 66,6$, y en fines de semana 94,1 $\pm 73,6$ gramos/día.

En la tabla 4 se recogen las características de la población que utiliza el recurso en la primera demanda de tratamiento en la UMD y las características de los que acuden al Centro de Atención al Drogodependiente de Murcia en el mismo período de tiempo.

\section{Utilización de la Unidad Móvil de Drogodependencias}

Acudir a la UMD era la primera demanda de tratamiento para 51 casos (37,8\%). Los pacientes habían acudido a la UMD informados en 71 casos por amigos $(52,6 \%), 25$ por familiares (18,5\%), 24 no los había informado nadie, los usuarios habían visto la UMD $(17,8 \%)$, 8 habían sido informados por conocidos $(5,9 \%)$ y 7 lo fueron en un centro $(5,2 \%)$. La UMD había sido utilizada en alguna ocasión por la persona que los había informado, lo cual sucedió en 100 casos $(74,1 \%)$. El informador seguía utilizando esta UMD en 95 casos $(71,1 \%)$. El paciente acude a la UMD por iniciativa propia en 97 casos (71,9\%) y acompañado por otro paciente en 38 ocasiones $(28,1 \%)$.

\section{Variables clínicas}

Finalmente por lo que respecta al conocimiento que tienen los pacientes sobre su estado serológico manifiestan ser seropositivos al $\mathrm{VIH}$ el 16,3\% de los sujetos, el $21,5 \%$ han tenido contacto con la hepatitis B, y el $20 \%$ con la hepatitis C, desconociendo su estado con relación a estas patologías más de un tercio de los usuarios. Aunque se observa un alto grado de desconocimiento, éste varía según la edad del paciente, así más del 50\% de los pacientes mayores de 30 años manifiestan conocerlo y no estar afectados, el $68,7 \%$ de los menores de 20 años desconocen cuál es su estado serológico respecto a estas enfermedades. En el mes previo a la entrevista el 30,3\% de los sujetos solicitaron asistencia en Atención Primaria, y el $26,5 \%$ acudieron a un servicio de urgencias $(22,1 \%$ 
por patología orgánica y el 4,4\% por síndrome de abstinencia.)

\section{DISCUSIÓN}

El perfil sociodemográfico de la población estudiada es semejante al encontrado por la mayoría de los autores consultados ${ }^{7-10}$. Se trata de varones jóvenes con una edad media de 26,2 años. Esta edad es inferior a otros estudios realizados en España, así como en otros realizados en poblaciones como la norteamericana u holandesa que está en torno a los 30 años ${ }^{10-}$ 14. El porcentaje de mujeres es sólo del 11,9\%, más bajo que el descrito por otros autores españoles $7,9,10,13$ (en los que se alcanza el 20\%), Ilegando al 30\% en ciudades como Berlín o Londres ${ }^{15}$.

Otra características de la población estudiada son el bajo nivel de escolarización, la presencia de historial delictivo y bajo nivel de empleo. Así solo el 27,4\% finalizó los estudios primarios, el 29,6\% de los usuarios de la UMD tenían antecedentes de contacto con el Juzgado de Menores y el $74,1 \%$ de los casos habían sido detenidos en alguna ocasión. En el año previo a la demanda de tratamiento en la UMD, un tercio de nuestros usuarios habían sido detenidos una media de dos veces, con al menos un ingreso en prisión, lo que puede sugerir la existencia de sujetos cuyo patrón de vida habitual está muy relacionado con la actividad delictiva. El motivo de detención más frecuentemente asociado al abuso de sustancias tóxicas lo constituye los delitos contra la propiedad ${ }^{16}$. Recientemente otros autores españoles también refieren el bajo nivel educativo, alto nivel de desempleo y de historial delictivo en sujetos en tratamiento en programas de mantenimiento con metadona ${ }^{10}$.

La asociación entre el consumo de drogas y la actividad delictiva ha sido ampliamente demostrada, aunque la naturaleza de esta asociación es controvertida. Kaye et al.. (1998) ${ }^{17}$ investigan pacientes sometidos a un programa de mantenimiento con metadona encontrando que el abuso/dependencia de opiáceos fue el factor de riesgo más importante para la reincidencia delictiva, fundamentalmente delitos contra la propiedad $(77,3 \%)$ frente a un grupo control $(20,1 \%)$. Estos resultados sugieren la relación entre el abuso/dependencia de opiáceos y delincuencia, probablemente basada en necesidades económicas. Sin embargo, esta asociación no parece tener una causa única y directa sino que otros factores parecen influir ${ }^{18}$.

Hemos comprobado una progresión en el consumo de las diferentes sustancias. El primer contacto se tiene con las drogas legales y suele ser el tabaco, con una edad media de inicio de 12,6 años, seguido del cannabis y del alcohol. Posteriormente se entra en contacto con los alucinógenos y las anfetaminas, quedándose en la mayoría de nuestros usuarios en el simple uso. Más tarde, se incorporan otras sustancias de efectos más potentes, como la heroína, cocaína y benzodiacepinas. Mayoritariamente los sujetos se caracterizan por el policonsumo, siendo la cocaína la droga secundaria de mayor consumo $(64,6 \%)$. Características similares se han descrito por otros autores en poblaciones en España ${ }^{10}$.

Con respecto a la situación $\mathrm{VIH}$, podemos destacar que, a pesar de la incidencia de la infección entre los usuarios de drogas, se siguen manteniendo porcentajes elevados de sujetos que demandan tratamiento, y que desconocen cual es su situación con respecto al VIH, bien por no haberse realizado nunca las pruebas o bien por desconocer el resultado de las mismas. Tan solo hemos podido llevar a cabo analíticas para el VIH en el $46,7 \%$ de los usuarios y en el $43 \%$ en el caso de la Hepatitis B y C. En el resto de los casos, nos encontramos ante una negativa, ya que en muchas ocasiones son muy reacios a tener conocimiento de su estado de salud, quizás por miedo a conocer los resultados, especialmente en el caso de la infección por el VIH.

Al realizarse la entrevista, comprobamos como un gran porcentaje de pacientes que se iniciaron por la vía intravenosa y los que siguen utilizándola compartieron jeringuillas, bien con una misma persona, como es el caso de las mujeres, o bien con diferentes personas. No obstante, llama la atención que en ningún caso se hubiese utilizado una jeringuilla de procedencia desconocida. La frecuencia de intercambio de jeringuillas con diferentes personas $16,2 \%$ de las mujeres y el $3,4 \%$ de los hombres) es una de las más bajas encontradas en la bibliografía, en la que se alcanzan porcentajes entre el $25 \%$ y $50 \%$. Bravo Portela et al.. ${ }^{19}$ encuentran que las prácticas de riesgo de inyección disminuyen fundamentalmente entre los infectados por el VIH, por lo que sería necesario promocionar la prueba del VIH y proporcionar consejo específico según el estado serológico y prácticas de riesgo ${ }^{20}$.

Con referencia al VIH los porcentajes encontrados en nuestra población, aunque muy poco elevados son preocupantes, sobre todo en el caso de las mujeres, por tratarse de una población fértil y que ejerce con frecuencia la prostitución. Teira et al.. ${ }^{21}$ confirman el crecimiento de la proporción de mujeres con diagnóstico reciente de VIH en España.

El patrón de consumo que caracteriza a nuestra muestra es la politoxicomanía. El 66,6\% de los sujetos consumen diariamente cocaína y también consumen a diario cannabis $(19,3 \%)$ y benzodiacepinas $(17,8 \%)$, junto con una media de 66,7 gr. de alcohol. Esta cantidad se eleva a 94,1 gr. en los fines de semana, lo que nos permite clasificarlos como bebedores excesivos. 
Este elevado consumo de alcohol ha sido también constatado en otros estudios y debería ser objeto de mayor atención por parte de los profesionales ${ }^{22,23}$.

En cuanto al perfil de los usuarios de etnia gitana que demandan tratamiento en la UMD coincide con el descrito por otros autores ${ }^{24,25}$. Se trata mayoritariamente de un hombre joven, casado o conviviendo con la pareja, con baja cualificación académica, que han sido detenidos a una edad precoz, pero no presentan diferencias en cuanto a antecedentes delictivos con el resto de pacientes estudiados. Estos pacientes son los que gastan más dinero en el consumo de heroína y cocaína, destacando además que son más higiénicos en el uso de la jeringuilla que el resto de los pacientes de nuestra muestra ya que mientras en éstos la mayoría utilizan la vía intravenosa los pacientes de etnia gitana la consumen inhalada/fumada. $Y$ en cuanto a la situación serológica de estos sujetos encontramos porcentajes similares a otros estudios ${ }^{24}$. Hay que señalar que en la comunidad gitana existe una notable ausencia de datos sobre la incidencia de VIH/SIDA cuyo diagnóstico se produce con cierto retraso en relación con la población general, teniendo una mayor resistencia a conocer la seropositividad, al tratamiento, al seguimiento y a que la familia conozca su situación. Así en nuestro estudio encontramos una resistencia en el 81,5\% de los casos a conocer cuál es su estado, respecto a estas infecciones. Es necesario establecer medidas informativas y preventivas adaptadas a la idiosincrasia de la cultura gitana ${ }^{26}$.

Los usuarios, en un determinado momento comienzan a tener conciencia de que su problema está relacionado con el consumo de drogas y solicitan tratamiento. En la actualidad al crearse este tipo de recursos, de reducción de riesgos, mucho más flexibles, comprobamos que a él acude una población que hasta ese momento no se había planteado acceder a un recurso terapéutico porque asumían previamente el fracaso. Estos programas permiten captar a esta población e intentar derivarla a otro tipo de tratamientos. En este sentido, dentro de nuestra muestra encontramos que el $37,8 \%$ de los pacientes era la primera demanda de tratamiento de su dependencia, siendo del $81,5 \%$ en los sujetos de la población gitana, por lo que se cumple uno de los objetivos para los que se crean este tipo de recursos, como es llegar a esta población que nunca ha sido tratada.

Una de las cuestiones más importantes, desde el punto de vista asistencial, es conocer el tipo de población que utiliza este tipo de recurso, y establecer las similitudes y/o diferencias con la población que acude a otros recursos. Los usuarios de la UMD se diferencian de los usuarios del Centro de Atención de Drogodependencias teniendo en general un perfil de mayor riesgo. A la UMD acuden un mayor porcentaje de policonsumidores, en los que la vía principal de consumo de la heroína es la intravenosa, con una mayor precocidad y años de consumo.

Aunque en el 71,4\% de los casos los usuarios acuden a la UMD por iniciativa propia, es de destacar la amplia proporción de pacientes en cuyo inicio de tratamiento influye la opinión de otro paciente. En el 90\% de los casos, la persona que informa de la UMD ha usado el servicio o lo sigue haciendo, lo que nos puede dar una idea del nivel de aceptación del servicio por los usuarios del mismo, destacando la gran influencia de los pares para iniciar el tratamiento.

En nuestra opinión, se confirma la necesidad de este tipo de recurso para aquellos consumidores de drogas caracterizados por la marginación social y actitudes de rechazo inicial a los programas terapéuticos tradicionales.

\section{REFERENCIAS}

1. Brugal MT, Barrio G, Royuela L, Bravo MJ, de la Fuente $L$, Regidor E. Estimación de la mortalidad atribuible al consumo de drogas ilegales en España. Med Clin (Barc) 2004; 123:775-7.

2. Aceijas C, Stimson GV, Hickman M, Rhodes T; United nations Reefernce Group on HIV/AIDS Prevention and Care among IDU in Developing and Transitional Countries. Global overview of injecting drug use and HIV infection among injecting drug users. AIDS 2004; 19:2295-303.

3. Hope VD, Judd A, Hickman M, Sutton A, Stimson GV, Parry JV, Gill ON. HIV prevalence among injecting drug users in England and Wales 1990 to 2003: evidence for increased transmission in recent years. AIDS 2005; 19: 1207-1214.

4. Ward J, Hall W, mattick RP. Role of maintenance treatment in opiod dependence. Lancet 1999; 353:221-6.

5. Rounsaville BJ, Kosten TR. Treatment for opioid dependence: quality and access. JAMA 2000; 283:1337-9.

6. Ward J, Mattick RP, Hall W. The efectiveness of methadone maintenance treatment: an overview. Drug and Alcohol Review 1994; 13:327-36.

7. Hernández-Aguado I, Bolumar F. Determinants of HIV infection in intravenous drug users in Valencia, Spain, 1987-1991. Int J Epidemiol 1993; 22:537-42.

8. Kang SY, Delon G. Correlates of drug injection behaviors among methadone outpatients. Am J Drug Alcohol Abuse 1993; 19:107-18.

9. Torrens M, San L, Martínez A, Castillo C, Domingo-Salvany A, Alonso J. Use of the Nottingham Health Profile for measuring health status of patients in methadone maintenance treatment. Addiction 1992; 92:707-16. 
10. Puigdollers E, Domingo-Salvany A, Brugal MT, Torrens M, Alvaros J, Castillo C, et al. Characteristics of heroin addicts entering methadone maintenance treatment: quality of life and gender. Subst Use Misuse 2004; 39:1353-68.

11. Cacciola JS, Alterman Al, Rutherford MJ, McKay JR, McLellan AT. The early course of change in methadone maintenance. Addiction 1998; 1:41-9.

12. Lee SS, Hollinrake JB, Ng MH. Changing behavioural pattern of drug users in Hong-Kong 1991-1995. Addiction 1998; 93:541-48.

13. Domingo-Salvany A, Perez K, Torrens M, Bravo MJ, Anto JM, Alonso J. Methadone treatment in Spain, 1994. Drug Alcohol Depend 1999; 56:61-6.

14. Perez-Gonzalez K, Domingo-Salvany A, Hartnoll R. The characteristics of opiate users seen in an emergency service. Gac Sanit 1999; 13:88-95.

15. World Health Organization. Programme on substance abuse. An international comparative study of HIV prevalence and risk behavior among drug injectors in 13 cities. Bull Narc 1993; 45:19-46.

16. Osuna E, Luna A. Behavior at school and social maladjustment. J Forensic Sci 1989; 34:1228-34.

17. Kaye S, Darke S, Finlay JR. The onset of heroin use and criminal behaviour: does order make a difference? Drug Alcohol Depen 1998; 53:79-86.

18. Morentin B, Callado LF, Meana JJ. Differences in criminal activity between heroin abusers and subjects without psychiatric disorders-analysis of 578 detainees in Bilbao, Spain. J Forensic Sci 1998; 43:993-9.

19. Bravo Portela MJ, Barrio Anta G, de la Fuente de Hoz L, Royuela Morales L, Colomo Gómez C, Rodríguez Arenas A. Evolution of the prevalence of HIV infection and risk behavior of drug injection among intravenous drug users infected or not infected by HIV in 3 Spanish cities. Working group of Physicians of the World for the monitoring of HIV infection and risk behaviors in intravenous drug users. Rev Clin Esp 2000; 200:355-9.

20. Gossop M, Marsden J, Stewart D, Treacy S. Reduced infection risk and sexual risk behaviours after drug misuse treatment: results from the National Tretament Outcome Research Study. AIDS Care 2002; 14:77-93.

21. Teira R, Suarez-Lozano I, Muñoz P, Viciana P, Lozano F, Galindo MJ, et al.. Patrón epidemiológico de la infección por el VIH en 15 hospitales españoles: casos de nuevo diagnóstico incluidos en la cohorte $\mathrm{VACH}$ en 2001 y 2002. Enferm Infecc Microbiol Clin 2005; 23:213-7.

22. Ruz Franzi I, Gonzalez-Saiz F, Ruiz Avila F. Drug dependent patients in the methadone maintenance program: evaluation in primary care of psychosocial and organic severity. Aten Primaria 1998; 21:384-8.

23. Backmund M, Schutz CG, Meyer K, Eichenlaub D, Soyka $\mathrm{M}$. Alcohol consumption heroin users, methadonesubstituted and codeine-substituted patientesfrecuency and correlates use. Eur Addiction Res 2003; 9:45-50.

24. Pereiro C, Pedreira JD, Castro A. Consumo de drogas e infección VIH/SIDA en individuos de etnia gitana. Salud y Comunidad gitana 1997; 1:14-6.

25. Jiménez Lerma JM, Iraurgi I, Zubizarreta Y, Murua F, Herrera A, Landabaso MA, Gutierrez-Fraile M. Características sociobiográficas y niveles de retención de un programa de antagonistas opiáceos en un colectivo gitano. Adicciones 1998; 10:23-31.

26. Iraurgi I, Jimenez-Lerma JM, Landabaso MA, Arrazola X, Gutierrez-Fraile M. Gypsies and drug addictions. Study of the adherence to treatment. Eur Addict Res 2000; $6: 34-41$. 